\title{
Bacterial culture of perfusion blood after open-heart surgery
}

\author{
R FREEMAN AND N HJERSING
}

From the Department of Microbiology, Freeman Hospital, Newcastle upon Tyne

ABSTRACT The results of routine culture of 595 consecutive specimens of perfusion blood are ò presented. Ten per cent of the specimens yielded bacteria overall, but it was found that the $e_{\circ}^{+}$ isolation rate was increased to $17.7 \%$ when the prophylactic antibiotics being given during the ${ }^{\supset}$ bypass were specifically neutralised. Coagulase-negative staphylococci and diphtheroids formed the majority of organisms isolated, but Gram negative bacilli of "coliform" type were also? occasionally found. A comparison of the relative findings in patients receiving prophylactic fluc-迎 loxacillin or cephradine showed that the isolation rates of coagulase-negative staphylococci and diphtheroids were lower in the group receiving flucloxacillin. The origin of the bacteria isolated $\infty$ from perfusion blood remains uncertain but speciation of coagulase-negative staphylococci from perfusion blood and similar organisms isolated subsequently from catheter tips in the sameo patients revealed no evidence that the two sources of organisms were linked. Although organisms are easily and commonly found in perfusion blood, the relevance of this phenomenon to postoperative endocarditis is not clear.

The most serious infective complication of openheart surgery is postoperative infection of the prosthetic material inserted at operation, a condition often referred to as postoperative endocarditis. The organisms associated with this infection are usually coagulase-negative staphylococci (Staphylococcus epidermidis, $S$ albus) and corynebacteria ("diphtheroids"). ${ }^{12}$ If the patient survives the operation by several months before acquiring endocarditis, the organisms then found to be causative agents are the classical organisms of endocarditis of natural valves, that is streptococci. ${ }^{2}$ This change in the causative organisms of endocarditis with the passage of time after the operation has been taken to indicate a particular susceptibility to infection of the prosthetic valve to coagulase-negative staphylococci, diphtheroids, and, occasionally, other unusual organisms in the operative and immediate postoperative periods. Many of the schemes of antibiotic prophylaxis used in open-heart surgery are based on this observation.

Central to this theme is the concept that coagulase-negative staphylococci and diphtheroids

Address for reprint requests: Dr R/Freeman, Department of Microbiology, Freeman Hospital, Freeman Road, High Heaton, Newcastle upon Tyne NE7 7DN. must gain access to the prosthesis at or immediately after operation. The finding of such organisms on the tips of catheters used in postoperative management has been documented, ${ }^{3}$ and may provide one source of these agents, but another source may be the perfusion apparatus, and the isolation of organisms from the extracorporeal 3 circulation has been well described. ${ }^{4}$ In this report ${ }_{0}$ we detail the findings obtained by routine culture of blood taken from the bypass machine in 595 consecutive patients undergoing open-heart surgery.

\section{Methods}

No selection of patients was attempted. At the $\tilde{\sim}$ end of perfusion $10 \mathrm{ml}$ of blood was withdrawn $\underset{\omega}{N}$ with aseptic precautions from the oxygenator and 0 added to a blood culture bottle containing $100 \mathrm{ml}$ of glucose broth. The blood cultures obtained $\underset{\mathbb{D}}{\overparen{D}}$ were transported to the laboratory for incubation $\stackrel{\mathcal{Q}}{\rightarrow}$ which was at $37^{\circ} \mathrm{C}$. The bottle was inspected after 0 overnight incubation for evidence of turbidity and

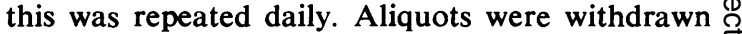
for culture at 24 hours and again after eight days' $\stackrel{\mathbb{D}}{\square}$ incubation. These intervals are referred to sub- $\overline{0}$ sequently as preliminary and later. Gram-stained films of the bottle contents were only per- 
formed when inspection revealed turbidity, in order to minimise the possibility of laboratory contamination.

All the patients studied were receiving routine antibiotic prophylaxis. In 489 instances this was flucloxacillin, administered as a large intravenous dose $(2 \mathrm{~g}$ in adult patients, proportionately less in children) at induction of anaesthesia. In 93 instances the antibiotic given was cephradine ( $1 \mathrm{~g}$ iv) and all these patients were adults. The remaining 13 patients received lincomycin $(600 \mathrm{mg}$ iv in adults, proportionately less in children) since they were allergic to penicillins. Although the prophylaxis was continued postoperatively in all cases, no further doses of antibiotics were given during perfusion.

In the first 448 instances no substance designed to neutralise the antibiotics was added to the culture, but in the last 147 instances an excess of Whatman II beta-lactamase (Whatman Biochemicals Ltd) was added to the cultures of those patients receiving flucloxacillin or cephradine.

Culture of aliquots from the blood cultures was carried out both aerobically and anaerobically and all organisms were identified by standard methods. In 23 cases the coagulase-negative staphylococci isolated were further subdivided using the API-staph system. Using this system it is possible to subdivide coagulase-negative staphylococci into several species, the main ones being Staphylococcus epidermidis, $S$ hominis, $S$ saprophyticus, $S$ hemolyticus, $S$ warneri, $S$ simulans, and $S$ capitis.

\section{Results}

As table 1 shows, $10 \%$ of the perfusion blood cultures yielded an organism, and coagulase- negative staphylococci and diphtheroids were the two most common organisms found. Whereas the isolations of coagulase-negative staphylococci were accomplished as commonly within 24 hours of the culture being drawn (preliminary) as on prolonged incubation (later), the diphtheroids were isolated more frequently after longer incubation. This slow growth pattern is typical of diphtheroids, and does not necessarily indicate laboratory contamination.

In table 2 the effect of using a beta-lactamase preparation is seen. The preparation used is known to inactivate flucloxacillin and cephalosporins. It is seen that the isolation rate of organisms is doubled $(8.3 \%$ to $17.7 \%)$. The increased isolation rate is due to increased numbers of coagulase-negative staphylococci and diphtheroids, whereas no increase in the isolation rates of the other organisms was found. Thus, the increase in isolations corresponds with the known spectrum of flucloxacillin.

Table 3 contains a comparison of results obtained when different antibiotics were being used for prophylaxis. The isolation rate using cephradine is higher than when flucloxacillin is being used, although this is a difference confined to coagulase-negative staphylococci and diphtheroids. In view of the fact that the spectrum of cephradine includes Gram negative facultatively anaerobic bacilli ("coliforms"), whereas that of flucloxacillin does not, it is interesting to note that such organisms were only found in the flucloxacillin group. From table 4 it can be seen that the vast majority of coagulase-negative staphylococci found were speciated as Staphylococcus epidermidis, but it is important to note that three isolates were not (two strains of Staphylococcus saprophyticus and one of Staphylococcus capitis). The correlation of these results with those ob-

Table 1 Overall isolation rate of various organisms from culturcs of perfusion blood, and distribution of the isolates with regard to the time of laboratory incubation. The apparent discrepancy between the number of positive cultures and the total number of organisms is caused by occasional cultures yielding more than one organism

\begin{tabular}{llllll}
\hline $\begin{array}{l}\text { Number of } \\
\text { cultures }\end{array}$ & $\begin{array}{l}\text { Number } \\
\text { positive }\end{array}$ & $\begin{array}{l}\text { Coagulase } \\
\text { negative } \\
\text { staphylococci }\end{array}$ & Diphtheroids & Streptococci & Coliforms* \\
595 & $63(10.6 \%)$ & $20(3.4 \%)$ & $7(1 \cdot 2 \%)$ & $3(0.5 \%)$ \\
\hline
\end{tabular}

Distribution of the various isolates with regard to time of laboratory incubation
Organism

Coagulase-negative Preliminary Late Total

staphylococci

Diphtheroids

Streptococci

Coliforms

Others

12

24

34

$\begin{array}{lrr}1 & 19 & 20 \\ 4 & 3 & 7\end{array}$

$\mathbf{3}$

20

7

* This term refers to aerobic or facultatively anaerobic Gram negative bacilli. The three isolates were, in fact, Proteus mirabilis, Klebsiella
aerogenes, and Alcaligenes faecalis.
†The two organisms in this group were Micrococcus luteus and Veillonella. 
Table 2 Effect of routine addition of a broad spectrum beta-lactamase preparation to cultures of perfusion blood

\begin{tabular}{|c|c|c|c|c|c|c|c|}
\hline \multicolumn{8}{|c|}{ Effect of adding beta-lactamase to perfusion blood cultures-overall isolation rate, and organism-specific isolation rates } \\
\hline Beta-lactamase & $\begin{array}{l}\text { No of } \\
\text { cultures }\end{array}$ & $\begin{array}{l}\text { No of positive } \\
\text { cultures }\end{array}$ & $\begin{array}{l}\text { Coagulase } \\
\text { negative } \\
\text { staphylococci }\end{array}$ & Diphtheroids & Streptococci & Coliforms & Others \\
\hline $\begin{array}{l}\text { Not added } \\
\text { Added }\end{array}$ & $\begin{array}{l}448 \\
147\end{array}$ & $\begin{array}{l}37(8 \cdot 3 \%) \\
26(17 \cdot 7 \%)\end{array}$ & $\begin{array}{l}20(4 \cdot 5 \%) \\
16(10 \cdot 8 \%)\end{array}$ & $\begin{array}{r}12(2 \cdot 7 \%) \\
8(5 \cdot 4 \%)\end{array}$ & $\begin{array}{l}5(1 \cdot 1 \%) \\
2(1 \cdot 4 \%)\end{array}$ & $\begin{array}{l}2(0.4 \%) \\
1(0.7 \%)\end{array}$ & $\begin{array}{l}0 \\
2(1.4 \%)\end{array}$ \\
\hline \multicolumn{8}{|c|}{$\begin{array}{l}\text { Effect of beta-lactamase addition on distribution of the isolates relative to time of laboratory incubation } \\
\text { Lactamase not added ( } 448 \text { cultures) }\end{array}$} \\
\hline & & Preliminary & Late & & & & \\
\hline Coagulase-negat & ve staphylococci & 7 & 13 & & & & \\
\hline Diphtheroids & & 0 & 12 & & & & \\
\hline Streptococci & & 2 & 3 & & & & \\
\hline Coliforms & & 2 & $\mathbf{0}$ & & & & \\
\hline Others & & 0 & 0 & & & & \\
\hline $\begin{array}{l}\text { Total } \\
\text { Lactamase addeo }\end{array}$ & (147 cultures) & \multicolumn{6}{|c|}{ Lactamase added (147 cultures) } \\
\hline \multirow{4}{*}{\multicolumn{2}{|c|}{$\begin{array}{l}\text { Coagulase-negative staphylococci } \\
\text { Diphtheroids } \\
\text { Streptococci }\end{array}$}} & Preliminary & Late & & & & \\
\hline & & 5 & 11 & & & & \\
\hline & & 1 & 7 & & & & \\
\hline & & 2 & $\mathbf{0}$ & & & & \\
\hline \multicolumn{2}{|l|}{ Coliforms } & 1 & $\mathbf{0}$ & & & & \\
\hline \multicolumn{2}{|l|}{ Others } & 0 & 2 & & & & \\
\hline \multicolumn{2}{|l|}{ Total } & $9(6 \cdot 1 \%)$ & $20(13.6 \%)$ & & & & \\
\hline
\end{tabular}

Table 3 Overall and organism-specific isolation rates of organisms from cultures of perfusion blood, analysed according to the prophylactic antibiotic given

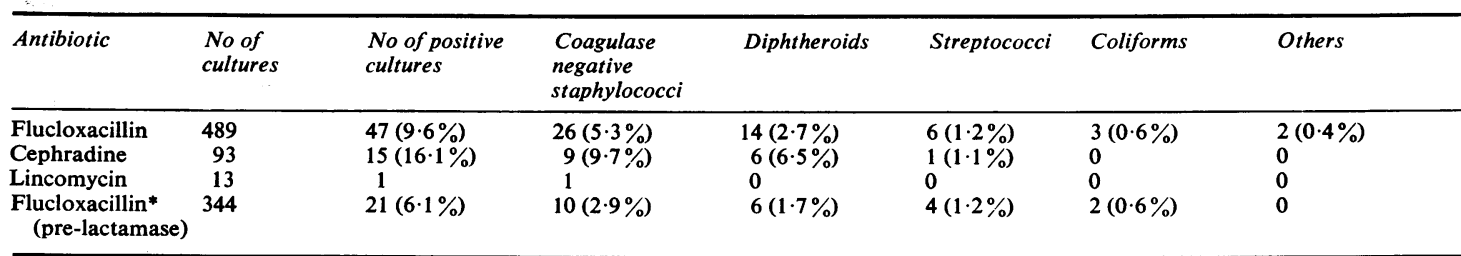

*The addition of beta-lactamase was introduced towards the end of the series, and no patient receiving cephradine occurred after its introduction This corrected figure for flucloxacillin thus allows a more valid direct comparison of these two agents.

Table 4 Analysis of the species of coagulase-negative staphylococcus isolated from cultures of perfusion blood, and their correlation with catheter tip cultures from the same patients. $(E=S$ epidermidis, $S=S$ saprophyticus, $C=S$ capitis $)$

\begin{tabular}{|c|c|c|c|c|c|c|c|}
\hline \multicolumn{6}{|c|}{ Results of speciation of 23 consecutive coagulase-negative staphylococci from perfusion blood cultures } & Incubaticn & Result \\
\hline 1 & Fluclos & Late & $\mathbf{E}$ & 13 & Fluclox & Late & $\mathbf{E}$ \\
\hline 2 & Fluclox & Preliminary & $\mathbf{E}$ & 14 & Fluclox & Late & $\mathbf{E}$ \\
\hline 3 & Fluclox & Late & $\mathbf{E}$ & 15 & Fluclox & Preliminary & $\overline{\mathbf{E}}$ \\
\hline 4 & Cephradine & Late & $\mathbf{E}$ & 16 & Fluclox & Late & $\mathbf{E}$ \\
\hline 5 & Cephradine & Late & $\mathbf{E}$ & 17 & Fluclox & Late & $\mathbf{E}$ \\
\hline 6 & Fluclox & Late & $\overline{\mathbf{S}}$ & 18 & Fluclox & Late & $\mathbf{E}$ \\
\hline 7 & Fluclox & Late & $\mathbf{E}$ & 19 & Fluclox & Preliminary & $\mathbf{E}$ \\
\hline 8 & Cephradine & Late & $\overline{\mathbf{E}}$ & 20 & Fluclox & Preliminary & $\mathbf{E}$ \\
\hline 9 & Lincomycin & Late & $\mathbf{S}$ & 21 & Fluclox & Late & $\mathbf{E}$ \\
\hline 10 & Fluclox & Preliminary & $\mathbf{E}$ & 22 & Fluclox & Late & C \\
\hline 11 & Fluclox & Late & $\mathbf{E}$ & 23 & Fluclox & Preliminary & $\mathbf{E}$ \\
\hline 12 & Fluclox & Late & $\mathbf{E}$ & & & & \\
\hline
\end{tabular}

Correlation of perfusion blood cultures and positive catheter tip cultures on 23 consecutive occasions

$\begin{array}{ll}\text { Number of patients Number of catheters Number positive } & \end{array}$

\begin{tabular}{ll} 
Positive cultures & \\
\hline Patient & Result \\
4 & S \\
6 & E \\
11 & S \\
22 & S
\end{tabular}


tained on culture of the catheter tips from the same patients is also seen. No evidence of identity of organisms was found.

\section{Discussion}

This series shows that routine culture of perfusion blood will regularly yield organisms. An important observation is that showing the efficacy of betalactamase addition, since, whatever the source of the organisms, it is clear that the incidence was being underestimated until this procedure was adopted. Earlier studies have also shown that similar organisms to the ones reported here have been isolated from perfusion blood. The two obvious points to be considered are the source(s) of the organisms and their relevance to the postoperative course of the patient, especially the incidence of postoperative endocarditis. The source of the organisms cannot be determined with any certainty from the present investigations. However, the organisms are predominantly those associated with the skin. It is reasonable, therefore, to suppose that they may be introduced as contaminants at the time of the culture being drawn. Thus, the origin might be the attendants taking the sample, or, alternatively the contaminants might be derived from the patient. Even if they were derived from the attendants taking the specimens, it is still not certain that they are without relevance to the patient since the site from which samples are taken might be the portal of entry for similar organisms which might result in endocarditis. The results of speciating the coagulase-negative staphylococci detailed in table 4 suggest that most of these organisms are of low significance if the results of earlier studies on catheter tip cultures are accepted. In catheter tip cultures it was found that $17 \%$ of the coagulasenegative staphylococci isolated were $S$ saprophyticus, and that this particular species was associated with catheters which had been in place for longer periods. ${ }^{5}$ In the present study of perfusion blood cultures, three of $23(13 \%)$ of the coagulasenegative staphylococci were other than $S$ epidermidis (two of 23 were $S$ saprophyticus). Earlier work on these patients suggests that $S$ epidermidis was likely to be a skin contaminant, whereas other species, especially $S$ saprophyticus, might be more pathogenic.

It is possible that the organisms detected in the perfusion blood might provide one source of the organisms subsequently found in the catheter tip cultures. While the limited survey shown in table 4 is insufficient to exclude this possibility, the failure to find any correlation between organisms found in the perfusion blood and catheter tips from the same patients suggest that any such correlation must be at best intermittent.

The question, therefore, of the relevance of these findings to the postoperative course of the patient must remain open. Of the 63 patients in whom the perfusion blood culture was positive, one has subsequently developed postoperative endocarditis, but the organism, a diphtheroid, was not recovered from the perfusion blood which yielded a coagulase-negative staphylococcus. However, this patient was studied early in the series, before beta-lactamase was added routinely. None of the patients in whom perfusion blood culture was negative has developed endocarditis.

Another point of note is the incidental comparison of flucloxacillin and cephradine. In the cephradine group, which is much smaller than the flucloxacillin group, a higher isolation rate was found of those organisms which both antibiotics act against (coagulase-negative staphylococci and diphtheroids). Thus, if the finding of organisms in perfusion blood cultures in any way measures the relative efficacy of the two drugs, it would seem that flucloxacillin is to be preferred. It is also, however, true that a small number of coliform organisms were found in the flucloxacillin group but not in the cephradine group, perhaps reflecting the Gram negative spectrum of the cephalosporin. Such organisms are not a common feature of postoperative endocarditis, and indeed one authority assesses the ability of them to cause the infection as very low compared to Gram positive organisms, ${ }^{6}$ and it would seem that this is a benefit of the cephalosporin which is outweighed by its inferior activity against the staphylococci and diphtheroids.

The conclusions to be drawn from this series are four. Firstly, organisms of the type which often cause postoperative endocarditis are easily recovered from perfusion blood. Secondly, the maximum yield of positive cultures is only obtained when the prophylactic antibiotic is specifically neutralised. Thirdly, the relevance of such findings is doubtful and, finally, it is likely that flucloxacillin is more effective in suppressing such organisms than cephradine. Further work is needed in order to assess the relevance of perfusion blood culture, and especially to determine the origin of the organisms isolated.

\section{References}

1 Johnson W D Jr. Prosthetic valve endocarditis. In: Kaye D (ed). Infective endocarditis. Baltimore: 
University Park Press, 1976; 129-42.

2 Dismukes WE, Karchmer AW, Buckley MJ et al. Prosthetic valve endocarditis: analysis of 38 cases. Circulation 1973; 48:65-377.

3 Freeman R, King B. Analysis of results of catheter tip cultures in open-heart surgery patients. Thorax 1975; 30:26-30.

4 Ankeney JL, Parker RF. Staphylococcal endocarditis following open-heart surgery related to positive intraoperative blood cultures. In: Brewer
LA (ed). Prosthetic heart valves. Springfield, Illinois: Charles C Thomas, 1969; 719-30.

5 Freeman $R$, Hjersing $H$. Species of coagulasenegative staphylococci isolated from catheter tips from open-heart surgery patients. Thorax $1980{ }^{\circ}$ 35:359-62.

6 Sande MA, Johnson WD Jr, Hook EW et al. Sustained bacteremia in patients with prosthetic cardiac valves. $N$ Engl J Med 1972; 286:1067-70. 